\title{
Spectrum sensing improvement in cognitive radio networks for real-time patients monitoring
}

\author{
Dramane Ouattara ${ }^{1}$, Francine Krief ${ }^{1}$, Mohamed Aymen Chalouf $^{2}$, and \\ Omessaad Hamdi ${ }^{1}$ \\ 1 Université de Bordeaux, LaBRI, 351 cours de la Libration \\ 33405 Talence Cedex, France \\ dramane.ouattara@labri.fr,francine.krief@labri.fr, ohamdi@labri.fr \\ 2 Université de Rennes 1, IRISA, IUT de Lannion, Rue Edouard Branly \\ 22300 Lannion, France \\ mohamed-aymen.chalouf@irisa.fr
}

\begin{abstract}
Regular monitoring of vital signs guarantees a preventive treatment of common diseases ensuring better health for people. Most of the proposed solutions in e-health context are based on a set of heterogeneous wireless sensors, fitting the patient and his environment. Often, these sensors are connected to a local smart node acting as a gateway to the outside (contacts, servers). When the patient is mobile, one of the issues we may face is the guarantee of a permanent connectivity between local smart node and the outside. To overcome this problem, we need to define a robust communications architecture able to benefit from different technologies and standards. This provides equipments with the ability to dispose of free-bands to perform their transmission anytime and anywhere. Cognitive radio, although appropriate technology, requires taking into account the interdependence between the patient's mobility and frequency band changes. Our proposal, is an anticipation model, a decision-making function that predicts the state of frequency bands occupancy. The model combines the machine learning techniques to the Grey Model system to provide low cost algorithm for spectral prediction which facilitates or guarantees permanent connectivity.
\end{abstract}

Key words: Cognitive radio networks, e-health, patients monitoring, connectivity, Grey Model, Machine Learning, spectral prediction.

\section{Introduction}

The emergence of new health risks, requires the design of new solutions able to assume a preventive role. These solutions must provide more autonomy to the patient with an anywhere and any-time monitoring capabilities. This requires a permanent connectivity and therefore, the spectral availability. In the context of communications rise in wireless networks, leading to the spectrum scarcity, cognitive radio is seen as a reliable alternative technology. However, remains a set of issues concerning spectrum sensing, spectrum sharing, spectrum decision and spectrum mobility to be solved for this technology. Concerning the spectrum 
mobility, issues related to permanent connectivity for mobile cognitive radio equipments are less explored. In this paper, we address the connectivity problem that could result from the patient's mobility. At this purpose, we propose a function that facilitates the detection of free-bands by the mobile cognitive radio equipment dedicated to the real-time patient's monitoring. This solution is based on machine learning techniques and the Grey Model system for performing a spectral prediction. The remainder of this paper is a definition of cognitive radio in section II, a state of the art on this technology used in e-health context in section III, the patients monitoring principle description in section IV , the proposed prediction model in section V. Finally, the section VI presents the experimental results and the section VII concludes.

\section{The cognitive radio networks}

\subsection{Definition and principle of cognitive radio networks}

The Cognitive Radio [1] is a paradigm for wireless networks where a node is able to automatically modify its transmitting parameters in order to communicate efficiently, while avoiding interference with other users. This self-configuration and self-adaptation of parameters is based on a set of modules and several factors in the internal or the external environment of the radio such as radio frequency, user behaviour and the network state.

\subsection{The modules of the cognitive radio}

Figure 1.1 summarizes the modules and the structure of the cognitive functions.

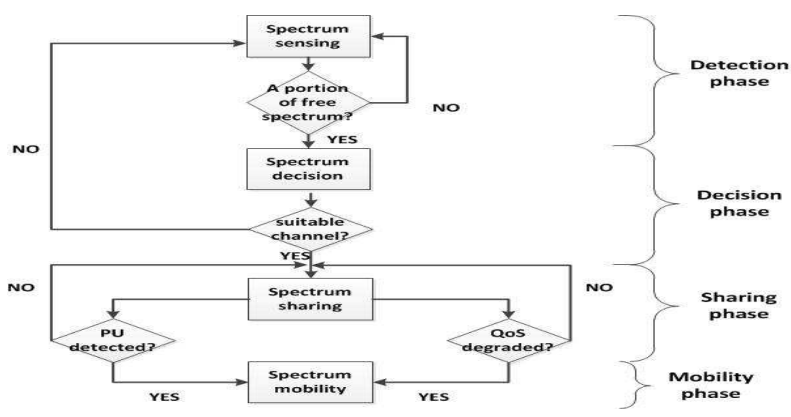

Fig. 1. Operating diagram of a cognitive radio node

The spectrum sensing : The spectrum sensing is defined as the ability to measure, examine, learn and be aware of the parameters related to the characteristics of the radio channel. This module measures the availability of spectrum, the signal strength, the interference and noise, scans operating environment of 
the radio, estimates the needs of the users and applications, checks the availability of networks and nodes, learn about the local policies and other operators restrictions.

The spectrum decision : The decision-making is based on the appropriate communication channel choice, justifying the quality of service required for the data or the collected information transmission.

The spectrum sharing : The channel sharing has to comply with the requirement of synchronized access to the detected free-bands portions. This scheduling is done between the secondary ${ }^{1}$ users on the one hand, and between these users and the primary ${ }^{2}$ users on the other.

The spectrum mobility : Spectrum mobility reflects the fact that each transceiver, must be able to change frequency band if the initial band becomes busy. Moving to a new frequency band could happen also when the initial band fails to provide the desired quality of service to the data transmission applications.

\section{Cognitive radio used in e-health context}

Cognitive radio technology used for patients monitoring is increasingly considered in the literature. An example of an e-health wireless communication system based on cognitive radio, deployed in a hospital setting is described in [2]. This article suggested answerers to the interferences caused by wireless transmissions to various medical devices. The proposal presented in [3], demonstrates the ability of cognitive radio to improve Wireless Body Area Networks (WBAN) performances. Another approach [4] proposes a parallel detection of vital signs and communication signals through the cognitive radio sensing module. From these different proposals and seen the cognitive radio technology capabilities, emerge the evidence, that dispose of a high channel availability by free-bands detection is essential for achieving the patients monitoring.

The patient monitoring requirements : The collected data by medical sensors followed by their transmission to a local or remote node are a range of services focused on communications. The emergency of medical data transmission and the need to have a robust communications architecture requires technologies that are able to provide a low cost communication links (bands) for ensuring reliable connectivity any-time and anywhere.

\section{The scenario addressed in patient monitoring}

Vital signs monitoring leads to data collecting by the medical sensors, a minimal treatment performed by the local smart node (fixed: Medical Box or mobile: Smart-phone, PDA) and a set of exchanges generated as shown at Figure 1.2.

\footnotetext{
${ }^{1}$ Cognitive radio users, who do not have any band-use license, such as the patient's equipments in our context.

${ }^{2}$ Users that have the band-use license.
} 


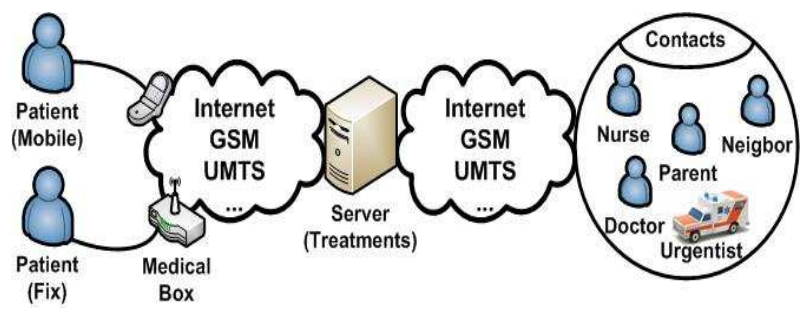

Fig. 2. The communication description scheme

Thus, the dissemination of the alerts and the medical data updates, increase demands of frequency bands. To ensure a continuous connectivity between the monitored patient in his environment and external actors (contacts, servers), the cognitive radio technology is well suited. According to the need for medical application, and depending on communication urgency and the frequency availability, the cognitive radio selects the appropriate channel, and the adequate technology to ensure transmissions. For this purpose, the communication protocol proposed in [5], based on a centralized architecture where a cognitive radio node (server) is responsible for the management of all transmissions (primary users and secondary users) by a queuing packets mechanism, provides partial response. In fact, this solution, is focused on the access synchronization to a single channel for the data transmission, explores the same technology and is limited to a hospital environment. Adapting this idea to our anywhere and any-time patient monitoring context, requires improvements to integrate multi-technologies and multichannel exploration capabilities. Thus, in addition to a distributed solution, our proposal offers an opportunity to explore all available technologies, to transmit on different channels and is adapted to the patient mobility context.

The spectrum sensing problematic: The spectrum sensing is the most expensive operation in terms of processing time and energy consumption for a mobile cognitive radio equipment. The energy autonomy to maximize for all mobile medical equipments can not tolerate an inefficient and inappropriate perpetual sensing. A spectrum prediction mechanism, measuring the channels states (free or occupied) probabilities, would significantly reduce the sensing frequency by ensuring a rational choice of the band to be sensed.

The limitations of existing prediction algorithms : The handover prediction is the most studied in the literature, leaving less-explored the spectral prediction in mobile environment. Spectral prediction studies using the game theory approaches and machine learning [6][7] are also proposed but in a static environment where there is no cell change. Others standard prediction model less considered in cognitive radio networks using the statistical techniques [8] such as AR (Auto Regressive), MA (Moving Average), ARMA (Auto Regressive Moving Average), ARIMA (Auto Regressive Integrated Moving Average) are also explored. However, these models require complete and representative data. The studies, whose aim is to reduce the cell change impact on connectiv- 
ity in cognitive radio networks by the design of prediction model, will ensure permanent transmissions required by the mobile patients monitoring process.

\section{The proposed solution}

The proposed solution is to add a prediction function to the spectrum sensing module as illustrated in Figure 1.1. This function combines the machine learning techniques with the Grey Model system to assess the probability of the channel occupancy and thus, guide the choice of the band to be sensed. Figure 1.3 represents a description of the prediction function operating mode and is more detailed in section 1.5.1. The goal is to have a low cost prediction algorithm with a learning module well trained but also compensate training time with Grey Model module predictions. This idea could enhance the current solutions, which consist in a set of multiple sensing to create free-bands data base. Our prediction proposal allows us to limit the number of sensing operations, and especially to achieve a timely sensing with significant reduction of computational cost.

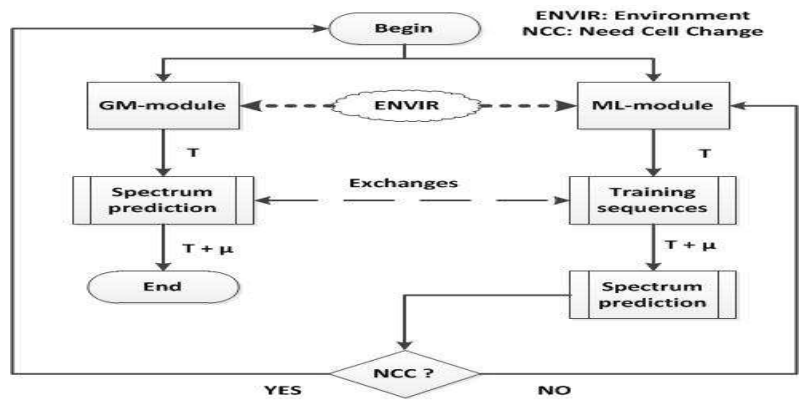

Fig. 3. Prediction function operating mode

\subsection{The details of the prediction process}

The Grey Model theory [9], is known for the analysis of problems with incomplete or uncertain information. Its main advantage on the statistical prediction techniques such as AR, MA, ARMA, ARIMA, is the ability to settle for a minimum input data to achieve its predictions. Our solution is based on the ability to predict, on the basis of incomplete data, provided by the Grey Model and the low cost processing offered by the machine learning algorithms when well trained. Indeed, the smart node runs with the two modules, Machine Learning (ML) and Grey Model (GM) in a new cell or at the beginning of its activity. The Grey Model at this stage makes predictions while the learning module executes training sequences. The prediction process returned to the ML-module, once its 
effectiveness is proved by the relevance of its prediction results and none Need of Cell Change (NCC) is detected as defined at figure 1.3.

\subsection{The model input data}

We are particularly interested to the $2.4 \mathrm{GHz}$ frequency band (WIFI). This is justified by characteristics of our platform GNU-Radio, but the principle is similar for the other channel types (GSM, Bluetooth, Zigbee etc.). Our goal being to achieve a probability distribution of the channel status on the future periods, leads to base the reasoning on the following parameters :

The energy on each sub-band : The energy detected on a channel could be used to identify a signal type [10]. A comparison of the received signal (energy) by the radio equipment to a predefined threshold depending on the type of channel, could determine a primary user signal status as shown at figure 1.4. The principle is based on two assumptions, $H_{0}(\mathrm{OFF})$ for channel is free and $H_{1}$ $(\mathrm{ON})$ for channel is busy such that $: H_{O}: y(t)=n(t)$ and $H 1: y(t)=x(t)+n(t)$ Where $\mathrm{t}=1,2, \ldots ., \mathrm{N}$ represents the received signal sample periods, $\mathrm{y}(\mathrm{t})$ the received signal, $\mathrm{n}(\mathrm{t})$ the Gaussian noise and $\mathrm{x}(\mathrm{t})$ the detected signal.

The other data : The other data to be taken into account are dynamic and static data. The dynamic data, much more random and scalable, includes energy, transmission power, number of primary users and number of free-channels. The static data can be defined as the number of channels, the band type, the location and the type of area (heavily urban, rural), the central frequency, the energy threshold.

The energy distribution on the channel : The patient's cognitive radio equipment makes periodic measurements of the channel energy. Our solution is a distribution of this data and its likelihood estimation on future periods.

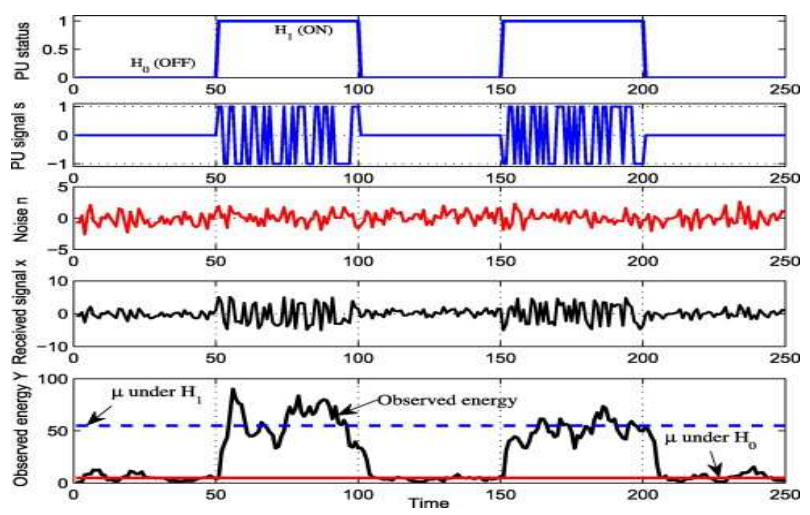

Fig. 4. Spectrum usage description

Figure 1.4 shows the links between the received signal and the energy of the band, the primary users signal distinction to Gaussian noise. the signal strength 
determines the presence $\left(H_{1} \mathrm{ON}\right)$ or the absence $\left(H_{0} \mathrm{OFF}\right)$ of a primary user. The mobile (Smart-phone) or the Medical Box equipment as described in figure 1.2 , exploits these periods (ON/OFF) for their medical data transmissions.

\subsection{Machine Learning and Grey Model description}

For the model simulation, we have opted for Hidden Markov Models (HMM) as learning module associated with the GM $(1,1)$. As objective, we have two quantities to optimize namely, minimize the cost and maximize the precision; Hence, the choice of GM for its reasonable cost and the HMM for its errorscorrecting ability.

The Hidden Markov Models (HMM) : The error-correcting technique is based on the observation of a trend curve and only the most recent values of the series are pertinent in predicting the future values. For a set of sampling data $\mathrm{X}(1), \mathrm{X}(2), \mathrm{X}(3), \ldots \ldots, \mathrm{X}(\mathrm{N})$, an adjustment function applied to the values, predicts the future trends by assessing weight to previous values as follow : $\operatorname{Xhat}(k)=p_{1} x_{k}+p_{2} x_{k-1}+p_{3} x_{k-2}+\ldots .+p_{n} x_{k-n+1}$, with $p_{1}, p_{2}, \ldots, p_{n}$, the weigths assigned to previous observations, where the most recent values have higher weight such as :

Xhat $(t)=\alpha *\left[x_{t-1}+(1-\alpha) x_{t-2}+(1-\alpha)^{2} x_{t-3}+(1-\alpha)^{3} x_{t-4}+\ldots\right]+(1-\alpha)^{t-1} x_{1}$ and $\alpha$ the adjustment coefficient, $\left(1,(1-\alpha),(1-\alpha)^{2},(1-\alpha)^{3},(1-\alpha)^{4}\right.$ the weights. The $\alpha$ value is thought of as a Markov chain which takes on a number of 'fuzzy' states. The m-step transition probabilities $\mathrm{p}(\mathrm{i}, \mathrm{j})$ of the chain are calculated by observing for each state i, the number of observed $\alpha$-values that drift state $\mathrm{j}$, in $\mathrm{m}$ quanta of time. The initial distribution of the chain is calculated by observing the number of points in the cluster(state). Given this initial distribution and the transition probabilities, the evolution of the chain is fully determined. Thus, the probability of transition from state $\mathrm{i}$ to state $\mathrm{j}$, denoted $P_{i j}$ is evaluated as follows : $P_{i j}=Q_{i j} / Q_{i}$, with $Q_{i}$, representing the number of times $\alpha$ remains in the state i and $P_{i j}$, the number of transitions occurrences between the state i and the state $\mathrm{j}$.

To the initial distribution is associated an initial vector : $\pi^{(0)}=\left[\pi_{1}^{(0)} \pi_{2}^{(0)} \pi_{3}^{(0)} \pi_{4}^{(0)} \pi_{5}^{(0)}\right]$, the transition probabilities. The probabilities distribution of future transition $(\mathrm{t}+1)$ states from $\pi^{(0)}$ is obtained by : $\pi^{(t+1)}=$ $\pi^{(0)} M^{(i)}$.

The Grey Model GM(1,1) : The GM $(1,1)$ is the most used in prediction systems [11].

\section{The GM(1,1) modelling process :}

- The system takes as input a sequence of values ( energy), with $X^{0}=$ $\left[x^{0}(1), x^{0}(2), x^{0}(3), \ldots, x^{0}(n)\right]$, the initial sequence, where $x^{0}(t)$ corresponds to the output of the system at time t.

- From the initial sequence, a new sequence $X^{1}$ is generated by the system with new values $X^{1}=\left[x^{1}(1), x^{1}(2), x^{1}(3), \ldots, x^{1}(n)\right]$.

- The first order differential equation obtained from $X^{1}$ is given by : $\frac{d x^{1}(t)}{d t}+$ $a x^{1}(t)=b, a$ is the coefficient that reflects the trend and $\mathrm{b}$ is the predic- 
tive control coefficient expressing the portion of the information known and unknown part of the information model.

- The parameter estimation or coefficients of the matrix $[a, b]^{T}$ can be obtained by the method of least squares knowing that $[a, b]^{T}=\left(B^{T} B\right)^{-1} B^{T} y_{N}$, where $B=\left[\begin{array}{cc}-\frac{1}{2}\left(x^{1}(1)+x^{1}(2)\right. & 1 \\ -\frac{1}{2}\left(x^{1}(2)+x^{1}(3)\right. & 1 \\ \ldots \ldots \ldots . & . \\ -\frac{1}{2}\left(x^{1}(n-1)+x^{1}(n)\right. & 1\end{array}\right]$

and $y_{N}=\left[x^{0}(2), x^{0}(3), x^{0}(4), \ldots, x^{0}(n)\right]^{T} ; \mathrm{n}$ being the size of the sequence defined by the model.

- The prediction function becomes : $\hat{x}^{1}(t)=\left(x^{1}(1)-\frac{b}{a}\right) e^{-a t}+\frac{b}{a}$

- The predicted value at time $t+1$ is obtained by : $\hat{x}^{0}(t+1)=\hat{x}^{1}(t+1)-\hat{x}^{1}(t)$.

\section{The experimentation}

Our study is based on an observation/analysis of the spectrum through cognitive radio platform and simulation results.

The cognitive radio platform : We set up a cognitive radio platform using GNU-Radio and the USRP1 of Ettus[12]. The ultimate goal is to transmit and receive through the platform, all radio standard (AM, FM, DAB, GSM, Wifi, GPS, TV) and also to analyse spectrum and medical signals.

The sample data and predicted values : On the basis of results described in [13], the ability of Grey Model to achieve predictions without any prior training (see figure 1.5) is proved. The simulation results (figure 1.6) are only the ML-module outputs. Based on the real-time measured values, adjustments are made by the learning function for taking into account the errors for future predictions. The values $(\mathrm{X})$ generated as our learning sample represent the energy on a sub-channel at different slot-time.

$\mathrm{X}=$\begin{tabular}{l|l|l|l|l|l|l|l|l|l|l|l|l|l|l|l|l|l|l|l|l|l|l|}
\hline 48 & 70 & 50 & 40 & 90 & 46 & 72 & 48 & 39 & 91 & 49 & 71 & 49 & 37 & 88 & 45 & 70 & 51 & 38 & 95 & 47 & 75 \\
\hline 50 & 36 & 93 & 42 & 76 & 60 & 40 & 98 & 41 & 77 & 55 & 39 & 97 & 42 & 73 & 50 & 38 & 99 & 41 & 76 & 53 & 37 \\
\hline
\end{tabular}

The predictions results :

Figure 1.6(a) shows that with adequate samples and training, the algorithm is able to control stationary events such as high activity moments of primary users and their low attendance times. The stationary behaviour of primary users allows obtaining better prediction results.

Figures 1.6(b) and (c) show that the behaviour of primary users may be random and non-stationary and require more training time and sample data. In this case, the objectivity of the predictions is limited to one predicted value $(t+1)$. The predicted values distribution for the future periods $(\geq t+2)$, becomes less and less relevant. These results reveal the importance of the GM-module as an alternative prediction tool during the ML-module training periods. 

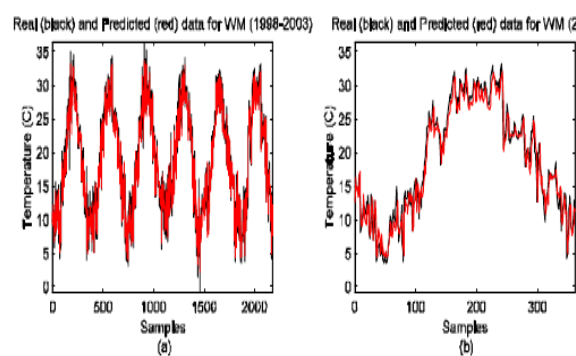

\begin{tabular}{|c|c|c|c|c|c|c|}
\hline \multicolumn{7}{|c|}{ 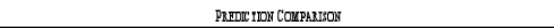 } \\
\hline Predictor & \multicolumn{4}{|c|}{ Fuzzy (WM) } & \multicolumn{2}{|r|}{ Grey } \\
\hline Inputs & $T(k-1)$ & $T(k-2)$ & $T(k-6)$ & $T(k-7)$ & $T(k-1)$ & \begin{tabular}{|l|l|l|}
$\mathrm{T}(\mathrm{k}-2)$ & $\mathrm{T}(\mathrm{k}-6)$ & $\mathrm{T}(\mathrm{k}-7)$ \\
\end{tabular} \\
\hline Mfs & 9 & 7 & 7 & 5 & \multirow{2}{*}{\multicolumn{2}{|c|}{$\begin{array}{c}\cdot \\
\mathrm{T}(\mathrm{k})\end{array}$}} \\
\hline Ouput & \multicolumn{4}{|c|}{$T(k)$} & & \\
\hline Mfs & \multicolumn{4}{|c|}{15} & \\
\hline $\begin{array}{c}\text { AND } \\
\text { Method }\end{array}$ & \multicolumn{4}{|c|}{ Product } & & \\
\hline Implication & & & & & \multirow{4}{*}{\multicolumn{2}{|c|}{. }} \\
\hline Aggregation & \multicolumn{4}{|c|}{ Sum } & & \\
\hline Defuzzifier & \multicolumn{4}{|c|}{ Centroid } & & \\
\hline Rules & \multicolumn{4}{|c|}{138} & & \\
\hline Results & \multicolumn{2}{|c|}{ Train } & \multicolumn{2}{|c|}{ Test } & & Local Prediction \\
\hline $\begin{array}{l}\text { Computation } \\
\text { Time }\end{array}$ & \multicolumn{2}{|c|}{$32.366 \mathrm{sec}$} & \multicolumn{2}{|c|}{$2.063 \mathrm{sec}$} & \multicolumn{2}{|r|}{$0.471 \mathrm{sec}$} \\
\hline AME & \multicolumn{2}{|c|}{1.385728} & \multicolumn{2}{|c|}{1.447687} & \multicolumn{2}{|r|}{1.407747} \\
\hline MSE & \multicolumn{2}{|c|}{3.066376} & \multicolumn{2}{|c|}{3.375785} & \multicolumn{2}{|r|}{3.524036} \\
\hline$p$ & \multicolumn{2}{|c|}{0.971563} & \multicolumn{2}{|c|}{0.972925} & \multicolumn{2}{|r|}{0.970235} \\
\hline $\begin{array}{l}\text { Maximum } \\
\text { Enror } \\
\text { (absolute) }\end{array}$ & \multicolumn{2}{|c|}{8.736815} & \multicolumn{2}{|c|}{8.198800} & \multicolumn{2}{|r|}{9.056811} \\
\hline
\end{tabular}
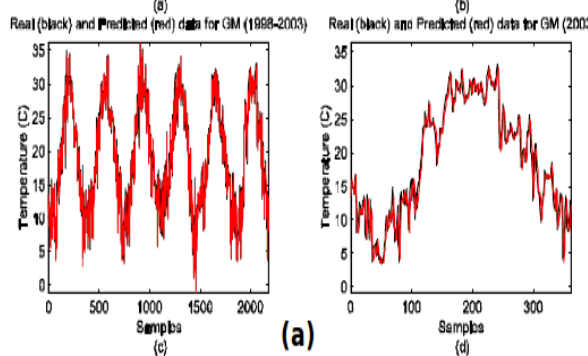

(b)

Fig. 5. Result of $\operatorname{GM}(1,1)$ predictions [13]
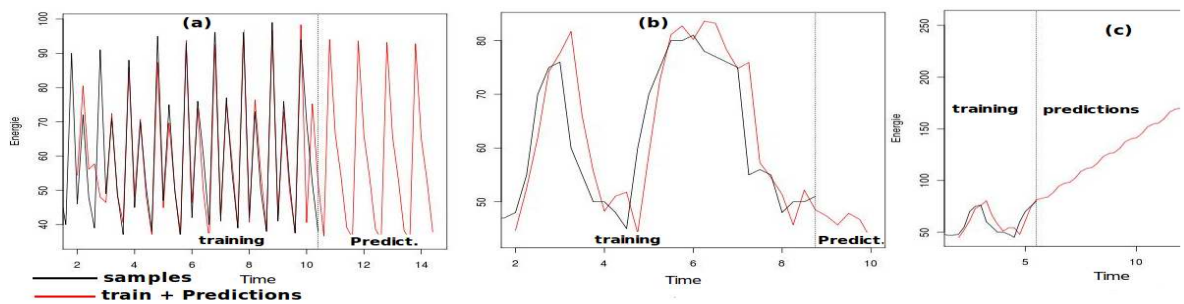

Fig. 6. Result of ML-module predictions

\section{Conclusion}

The problems for achieving the anywhere, any-time and real-time monitoring of vital signs, call for development of ambitious technologies able to overcome any lack of connection. In this paper, we have presented the concept of cognitive radio networks, seen as a solution to ensure constant connectivity for patient monitoring. We have also proposed a module, whose implementation would improve the sensing process for cognitive radio networks, crucial for the ongoing transmission of medical data. The spectral prediction model that we propose combines the Grey Model techniques to machine learning technology to ensure connectivity and facilitate channel change with low processing cost. Finally, we performed simulations based on machine learning to evaluate the prototype. The development of the Grey Model module for measuring the real performance of the complete model is part of our future works. 


\section{References}

1. J. Palicot. : Cog. Radio: An Enabling Technology for the Green Radio Communications Concept, Leipzig, Germany June 21 - 24, (2009)

2. P., P. and Hos., E. and Ni.: A cognitive radio system for e-health applications in a hospital environment : vol.17 : pp.20-28,(2010)

3. Feng Ji. and Liu Wei and Li Yang : Performance Enhancement of Wireless Body Area Network System Combined with Cognitive Radio : Vol3, pp.313-317, (2010)

4. F. Wang and C. Li and C. Hsiao : (MTT), IEEE MTT-S Int. : An injection-locked detector for concurrent spectrum and vital sign sensing : (2010),pp.768-771

5. Phun., P. and Hossain, E. and Niyato, IEEE : A cognitive radio system for e-health applications in a hospital environment : vol.17, pp.20-28, february (2010)

6. Y. Li and Y. Dong and Hui Z., IEEE 10th Intern. Conf. : Spectrum Usage Prediction Based on High-order Markov Model for Cognitive Radio Networks: july (2010)

7. Y. Liu and T., B.R. and Manoj, IEEE Global Telecom. Conf. : On Cognitive Network Channel Selection and the Impact on Transport Layer Performance : pp.1-5, Dec. (2010)

8. Xu Ye : G. Comput. (GrC), IEEE Intern. Conf. : The Application of ARIMA Model in Chinese Mobile User Prediction : pp.586-591, Aug.(2010)

9. Deng Julog : The Basis of Grey Theory : Huazhong University of Science and Technology Press, In Chinese, (2002)

10. Zhang Ling-ling and Huang Jian-guo and Tang Cheng-kai : Sig. Proc., IEEE Int. Conf. : Novel energy detection scheme in cognitive radio : pp.1-4, sept., (2011)

11. Chen S. and Ye Lin and Zhang G. and Zeng C. and Dong S. and Dai Chao : Short-term wind power prediction based on combined grey-Markov model : (2011), pp.1705 -1711, Vol.3

12. http://gnuradio.org/redmine/projects/gnuradio/wiki

13. A. I. Dounis and D. Tseles and G. N. : A Comparison of Grey Model and Fuzzy Predictive Model for Times Series : 2006, pp.176-181 